\title{
Communications
}

\section{Hydroarylation for the Facile Synthesis of 2-Substituted Tetrahydroquinoline: A Concise Synthesis of $(+)-(S)$-Angustureine}

\author{
Jae-Sang Ryu \\ College of Pharmacy, Ewha Womans University, Seoul 120-750, Korea.E-mail: ryuj@ewha.ac.kr \\ Received February 16, 2006
}

Key Words : Hydroarylation, Tetrahydroquinoline, $(+)-(S)$-Angustureine

Tetrahydroquinolines constitute important structural features present in a number of biologically active alkaloids. Especially, 2-substituted tetrahydroquinoline ${ }^{1}$ has drawn medicinal chemists' attention as a privileged structure. Angustureine, one member of 2-substituted tetrahydroquinoline alkaloids, was first isolated ${ }^{2}$ by JacquemondCollet and his co-workers in 1999 from Galipea officinalis, which has been used in traditional herbal medicine to treat a fever of dyspepsia, dysentery and chronic diarrhea. ${ }^{3}$ Recently, anti-tuberculous, ${ }^{4}$ anti-malarial, ${ }^{5}$ and cytotoxic ${ }^{5}$ activities have been reported for angustureine.

In the context to develop efficient synthetic methods for diversity oriented synthesis of tetrahydroquinolines, herein, we report a concise synthesis of $(+)-(S)$-angustureine and hydroarylation strategy. As outlined in Figure 1, our synthetic stratagem includes the introduction of a chiral side chain by Mitsunobu reaction ${ }^{6}$ and the subsequent hydroarylation to dihydroquinoline. This approach is flexible and applicable to the preparation of other 2-substituted tetrahydroquinolines, as well. To this end, we chose the aniline as our starting point (Scheme 1). $N$-methanesulfonyl protection of aniline, followed by Mitsunobu inversion ${ }^{6}$ of the $(R)-(+)-$ 1-octyn-3-ol (4) (98\% ee) with the resulting methanesulfonanilide 3 in the presence of DEAD/ $\mathrm{PPh}_{3}$, afforded $N$ propargylaniline 5. The Mesyl-NH group served as an efficient nucleophile for Mitsunobu reaction as well as an arene-free protecting group in the next hydroarylation step.

With the hydroarylation precursor $\mathbf{5}$ in hand, we have explored the feasibility of intramolecular hydroarylation under a variety of catalytic conditions (Table 1). We first

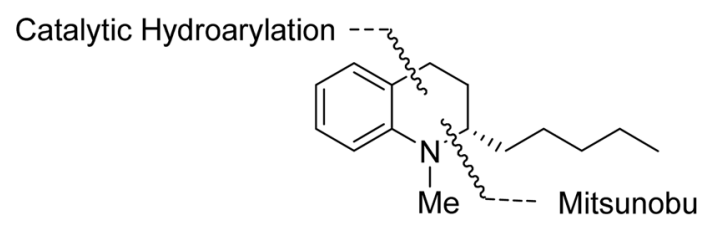

(+)-(S)-angustureine (1)

Figure 1. Key Features in Synthesis of $(+)-(S)$-Angustureine.
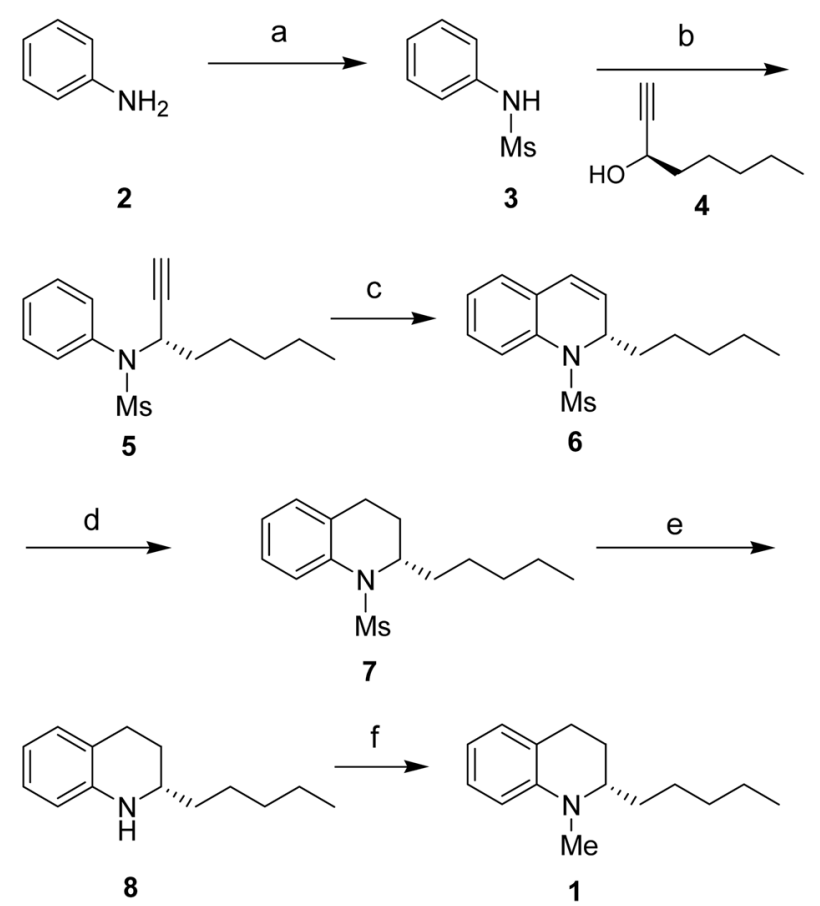

Scheme 1. Synthesis of (+)-(S)-Angustureine (1). (a) $\mathrm{MsCl}$, Pyridine, $\mathrm{CH}_{2} \mathrm{Cl}_{2}, 0{ }^{\circ} \mathrm{C}, 1 \mathrm{~h}, 92 \%$; (b) $(R)-(+)-1-$ Octyn-3-ol (4), DEAD, $\mathrm{Ph}_{3} \mathrm{P}, \mathrm{THF}, \mathrm{rt}, 1 \mathrm{~h}, 100 \%$; (c) See table 1; (d) $\mathrm{H}_{2}, \mathrm{Pd} / \mathrm{C}$, EtOH, rt, 3 h, 85\%; (e) Red-Al, toluene, $80{ }^{\circ} \mathrm{C}, 0.5$ h, 99\%; (f) $\mathrm{K}_{2} \mathrm{CO}_{3}$, THF, $\mathrm{CH}_{3} \mathrm{I}$, reflux, $24 \mathrm{~h}, 99 \%$.

tested the reaction with $\mathrm{AuCl}_{3}$ in the presence and absence of AgOTf. ${ }^{7}$ The catalysts were not active enough to complete the reaction within an acceptable reaction condition $(80$ ${ }^{\circ} \mathrm{C}, 18 \mathrm{~h}$ ) (entries 1 and 2$) \cdot \mathrm{Hg}(\mathrm{OTf})_{2}-(\mathrm{TMU})_{3}$ complex, ${ }^{8}$ which was reported as an efficient catalyst for the cyclization of activated arylalkynes, produced a unidentified byproduct as a major product with a small amount of dihydroquinoline 6 (entry 4). Then, we investigated platinum catalysts. We were pleased to find that $\mathrm{PtCl}_{4}$ was an effective catalyst to provide dihydroquinoline $6^{9}$ in a respectable yield (entry 8). None of undesired exomethylene regioisomer or $4 \mathrm{H}$-dihydroquinoline was detected. Generally, the hydroarylation of unactivated arylpropargylamine, especially 
Table 1. Catalytic Hydroarylation of $N$-propargyl methanesulfonanilide $\mathbf{5}$

\begin{tabular}{clccc} 
& & & \\
& & & \\
\hline
\end{tabular}

$\overline{{ }^{a} \text { Isolated yield. }{ }^{b} \text { Remaining starting material was recovered. }{ }^{c} \mathrm{~A} \text { unidenti- }}$ fied byproduct was isolated as a major product.

terminal alkynes, suffers from low activities. ${ }^{10}$ Although $\mathrm{Au}(\mathrm{III}),{ }^{7} \mathrm{Hg}(\mathrm{II}),{ }^{8}$ and $\mathrm{Pt}(\mathrm{II}){ }^{11}$ provided a few examples of activated-arylpropargylamine hydroarylations, the result with the unactivated substrate $\mathbf{5}$ was not satisfactory. To our best knowledge, our result constitutes the first example of a catalytic hydroarylation with unactivated $N$-propargylaniline to provide dihydroquinoline effectively. ${ }^{11}$

Reduction of the dihydroquinoline 6 was effected in $85 \%$ yield under standard catalytic hydrogenation conditions $(5 \%$ $\mathrm{Pd} / \mathrm{C}, \mathrm{EtOH})$. Removal of Ms protecting group of 7 was best achieved using Red-Al in toluene, ultimately affording the tetrahydroquinoline 8 in $99 \%$ yield. Finally, $N$-methylation completed the synthesis of $(+)-(S)$-angustureine. The spectroscopic data ${ }^{12}$ measured from 1 are in full accord with the published data ${ }^{13}$ of the compound.

In conclusion, we have accomplished a concise six-step synthesis of $(+)-(S)$-angustureine in overall $55 \%$ yield. The key features include an introduction of a chiral side chain by Mitsunobu reaction and an efficient Pt-catalyzed hydroarylation to dihydroquinoline. Given the result described above, research to expand hydroarylation into diversity oriented synthesis is currently in progress.
Acknowledgement. This work was supported by the Ewha Womans University Research Grant of 2005.

\section{References and Notes}

1. Katritzky, A. R.; Rachwal, S.; Rachwal, B. Tetrahedron 1996, 52, 15031.

2. Jacquemond-Collet, I.; Hannedouche, S.; Fabre, N.; Fourasté, I.; Moulis, C. Phytochemistry 1999, 51, 1167.

3. Mester, I. Fitoterapia 1973, 44, 123.

4. Houghton, P. J.; Woldemariam, T. Z.; Watanabe, T.; Yates, M. Planta Med. 1999, 65, 250.

5. Jacquemond-Collet, I.; Benoit-Vical, F.; Mustofa; Valentin, A.; Stanislas, E.; Mallié, M.; Fouraste, I. Planta Med. 2002, 68, 68.

6. (a) Mitsunobu, O.; Jenkins, I. D. In Encyclopedia of Reagents for Organic Synthesis; Paquette, L. A., Ed.; Wiley: New York, 1995; Vol. 8, p 5379. (b) Mitsunobu, O. Synthesis 1981, 1.

7. Shi, Z.; He, C. J. Org. Chem. 2004, 69, 3669.

8. Nishizawa, M.; Takao, H.; Yadav, V. K.; Imagawa, H.; Sugihara, T. Org. Lett. 2003, 5, 4563.

9. Representative procedure for $\mathrm{PtCl}_{4}$-catalyzed hydroarylation to dihydroquinoline 6: A solution of $N$-propargyl methanesulfonanilide 5 (28 mg, $0.1 \mathrm{mmol})$ in anhydrous dichloroethane $(1 \mathrm{~mL}, 0.1 \mathrm{M})$ was added to $\mathrm{PtCl}_{4}(3.4 \mathrm{mg}, 0.01 \mathrm{mmol})$. The reaction reaction mixture was stirred at $70{ }^{\circ} \mathrm{C}$ for $2 \mathrm{~h}$ under $\mathrm{N}_{2}$. The solvent was then removed in vacuo. Column chromatography on silica gel $(6: 1$ hexane/EtOAc) afforded pure dihydroquinoline 6 (20 mg; $72 \%$ yield). TLC: $R_{f} 0.27\left(6: 1\right.$ hexane/EtOAc). $[\alpha]_{\mathrm{D}}^{25}=$ $+287(c 1.0, \mathrm{MeOH}) .{ }^{1} \mathrm{H}-\mathrm{NMR}\left(400 \mathrm{MHz}, \mathrm{CDCl}_{3}\right): \delta 7.61(\mathrm{~d}, 1 \mathrm{H}$, $J=7.2$ ), 7.27 (td, $1 \mathrm{H}, J=7.2,1.6), 7.22$ (td, $1 \mathrm{H}, J=7.2,1.6$ ), 7.14 (dd, $1 \mathrm{H}, J=7.2,1.6), 6.53$ (d, $1 \mathrm{H}, J=9.6), 6.08(\mathrm{dd}, 1 \mathrm{H}, J=9.6$, 5.6), $4.72(\mathrm{~m}, 1 \mathrm{H}), 2.65(\mathrm{~s}, 3 \mathrm{H}), 1.49-1.34(\mathrm{~m}, 4 \mathrm{H}), 1.32-1.17(\mathrm{~m}$, $4 \mathrm{H}), 0.85(\mathrm{t}, 3 \mathrm{H}, J=7.2) .{ }^{13} \mathrm{C}-\mathrm{NMR}\left(100 \mathrm{MHz}, \mathrm{CDCl}_{3}\right): \delta 133.1$, $130.1,128.6,128.5,127.8,126.9,126.8,125.0,55.4,37.7,33.2$, $31.5,25.0,22.6,14.2$. HRMS (FAB): calcd for $\mathrm{C}_{15} \mathrm{H}_{22} \mathrm{NO}_{2} \mathrm{~S}$ $\left([\mathrm{M}+\mathrm{H}]^{+}\right), 280.1371$; found, 280.1374 .

10. Williamson, N. M.; Ward, D. A. Tetrahedron 2004, 61, 155.

11. Pt-catalyzed hydroarylation of arylpropargylether to chromene has been extensively studied by Sames. There are a few examples of arylpropargylamines activated by electron-donating groups, see: (a) Pastine, S. J.; Youn, S. W.; Sames, D. Org. Lett. 2003, 5, 1055. (b) Pastine, S. J.; Youn, S. W.; Sames, D. Tetrahedron 2003, $59,8859$.

12. TLC: $R_{f} 0.53\left(8: 1\right.$ hexane/EtOAc). $[\alpha]_{\mathrm{D}}^{25}=+6.1\left(c 1.0, \mathrm{CH}_{2} \mathrm{Cl}_{2}\right)$, $\left\{\right.$ lit. $\left.[\alpha]_{\mathrm{D}}^{26}=+4.4\left(c 1.0, \mathrm{CH}_{2} \mathrm{Cl}_{2}\right)\right\} .{ }^{1} \mathrm{H}-\mathrm{NMR}\left(400 \mathrm{MHz}, \mathrm{CDCl}_{3}\right)$ : $\delta 7.08(\mathrm{t}, 1 \mathrm{H}, J=7.2), 6.97$ (d, $1 \mathrm{H}, J=7.2), 6.58(\mathrm{t}, 1 \mathrm{H}, J=7.2)$, $6.53(\mathrm{~d}, 1 \mathrm{H}, J=7.2), 3.24(\mathrm{~m}, 1 \mathrm{H}), 2.93(\mathrm{~s}, 3 \mathrm{H}), 2.81(\mathrm{~m}, 1 \mathrm{H})$, $2.66(\mathrm{td}, 1 \mathrm{H}, J=16.0,4.0), 1.89(\mathrm{~m}, 2 \mathrm{H}), 1.63-1.27(\mathrm{~m}, 8 \mathrm{H}), 0.90$ (t, $3 \mathrm{H}, J=7.2) .{ }^{13} \mathrm{C}-\mathrm{NMR}\left(100 \mathrm{MHz}, \mathrm{CDCl}_{3}\right): \delta 145.6,128.8$, 127.3, 122.1, 115.4, 110.6, 59.2, 38.2, 32.2, 31.4, 26.0, 24.6, 23.8, 22.9, 14.2. HRMS (FAB): calcd for $\mathrm{C}_{15} \mathrm{H}_{23} \mathrm{~N}\left(\mathrm{M}^{+}\right), 217.1830$; found, 217.1832 .

13. Theeraladanon, C.; Arisawa, M.; Nakagawa, M.; Nishida, A. Tetrahedron: Asymmetry 2005, 16, 827. 